\title{
Delayed unilateral hematoma after reconstructive and aesthetic breast surgery with implants in Asian patients: two case reports
}

\author{
Jong Ho Lee ${ }^{1}$, Hyun Ki Hong ${ }^{1}$, Won Hwa Kim³ ${ }^{3}$ Hye Jung Kim ${ }^{3}$, Jeeyeon Lee ${ }^{2}$, Ho Yong Park ${ }^{2}$, \\ Jung Dug Yang', Joon Seok Lee ${ }^{1}$ \\ ${ }^{1}$ Department of Plastic and Reconstructive Surgery, School of Medicine, Kyungpook National University, Daegu, Korea; ${ }^{2}$ Department of Surgery, \\ School of Medicine, Kyungpook National University, Daegu, Korea; ${ }^{3}$ Department of Radiology, School of Medicine, Kyungpook National \\ University, Kyungpook National University Chilgok Hospital, Daegu, Korea \\ Correspondence to: Joon Seok Lee, MD, PhD. Department of Plastic and Reconstructive Surgery, School of Medicine, Kyungpook National \\ University, Kyungpook National University Chilgok-Hospital, 807, Hoguk-ro, Buk-gu, Daegu 41404, Korea. Email: leejspo@knu.ac.kr.
}

\begin{abstract}
Hematomas represent one of the postoperative complications in patients undergoing reconstructive or aesthetic breast surgery with a silicone implant. Although there are few reports of intracapsular hematoma, those presenting late hematoma after reconstructive and aesthetic augmentation surgeries are rarer. This study reported two Asian patients with late hematoma after reconstruction and aesthetic breast surgery. A 54-year-old female patient underwent bilateral nipple-sparing mastectomy with immediate breast reconstruction using anatomically shaped textured implant for intraductal carcinoma in August 2019. Contralateral nipple-sparing mastectomy was performed for the BRCA gene mutation on the left breast, which was immediately reconstructed with an anatomically shaped textured implant. In a 1-year postoperative magnetic resonance imaging evaluation, an extracapsular hematoma was found on the right side, which was removed following the removal of both implants. Another case was a 63 -year-old female patient who underwent augmentation of both breasts with smooth round implants and experienced right unilateral swelling and painless firmness about 30 years postoperatively. A preoperative magnetic resonance imaging evaluation showed both intracapsular and extracapsular ruptures on the right breast and a bulging implant herniation on the left breast. During the operation, hematoma, implants, and capsule were all removed. The excised capsule was sent for histological evaluation. Slightly dark colored blood was emptied before removing the semisolid-state intracapsular hematoma. In both cases, the patients responded well postoperatively and were discharged to their homes with no postsurgical complications, including seroma, or additional hematoma on the breasts. The etiology of late hematoma following breast augmentation or reconstruction has been poorly characterized. Further reports are needed to clearly establish the reasons for this increase in late hematoma formation.
\end{abstract}

Keywords: Hematoma; breast implantation; implant capsular contracture; hematoma post breast reconstruction; intracapsular hematoma; extracapsular hematoma

Submitted Nov 26, 2020. Accepted for publication Mar 05, 2021.

doi: $10.21037 / g s-20-854$

View this article at: http://dx.doi.org/10.21037/gs-20-854

\section{Introduction}

In Asian women with small- to moderate-sized breast, there is an increased demand for aesthetic breast augmentation, accompanied with popular immediate breast reconstruction using breast prosthesis after skin-sparing mastectomy in breast cancer cases. Except for skin cancer, breast cancer represents the most common cancer in women. The current protocol for breast cancer is immediate breast reconstruction rather than delayed one, and implant insertion is the recent preference among several reconstruction methods. Breast implantation, however, may 
lead to both early and late complications. Early hematomas remain one of the most common complications along with capsular contracture and seroma formation, whereas capsular contracture, implant rupture, and asymmetry are the most common delayed complications (1). A welldocumented complication among these complications is the early hematoma of a breast implant, occurring in $0-2 \%$ of patients, whereas delayed hematoma ( $>6$ months postoperatively) is much less common (2). Early hematomas frequently present within the first week after surgery, with significant pain and breast enlargement. They may occur more often in those who have taken vitamin E, estrogens, or aspirin or ibuprofen, both of which are known blood thinners. It is also more common in patients with elevated blood pressure or any sort of bleeding or clotting disorder, such as hemophilia. Overall, the mechanism of early hematoma includes failure of postoperative hemostasis. Late hematoma, however, is much less common, thus, this study aimed to establish the incidence of breast hematomas after implant placement and to identify risk factors to better characterize their occurrence. In this report, we present two rare cases of breast prosthesis implantation in Asian patients showing delayed unilateral extracapsular hematoma for reconstructive and aesthetic purposes. The two cases presented hereinafter have clinical significance in that the characteristics of hematoma in the preceding magnetic resonance image varied depending on the presence of breast parenchyma and whether the patients under went postoperative follow-up evaluations (e.g., mammography) between two cases.

This study has been conducted in accordance with the CARE reporting checklist (available at http://dx.doi. org/10.21037/gs-20-854).

\section{Case presentation}

\section{Case 1}

In August 2019, a 54-year-old woman underwent a bilateral nipple-sparing mastectomy via a single linear incision from the nipple to axilla for excising intraductal carcinoma presented on the left side in July 2019. Contralateral mastectomy was performed for prophylactic purposes toward the BRCA gene mutation. For immediate reconstruction, anatomically shaped macro-textured type, 415 and $370 \mathrm{cc}$ implants (BellaGel, HansBiomed Co. Ltd., Seoul, Korea) were used on the right and left breast, respectively. Skin-sparing mastectomy and prophylactic mastectomy on the left and right breasts were performed followed by implant insertion of different profiles. The remnant depth of skin flap, breast width, and mastectomy weight were also considered, which was 505 and $257 \mathrm{~g}$ for the left and right breast, respectively. The implant was inserted in the prepectoral plane, as first reported in the mid-1960s. Two kinds of acellular dermal matrix were wrapped around each implant in a double-crossed acellular dermal matrix technique (3). After 10 months postoperatively, the right breast had slowly developed significant evidence of Baker grade III capsular contracture as diagnosed by physical examination during clinical followup and associated with mild discomfort (Figure 1). Stiffness was observed throughout the right breast without pain during physical examination. The patient did not recall any event of trauma or external force toward the right breast, although mammography between postoperative 3 and 6 months could have inferred trauma, causing extracapsular hematoma on the right breast. Magnetic resonance imaging of the breasts demonstrated heterogeneous fluid collection on the retrocapsular area of the right breast, accompanied with linear leakage assuming extracapsular rupture and vessel erosion originating from the implant capsule (Figure 2). Preoperative lab finding including complete blood count, prothrombin time, metabolic panel, liver panel and inflammatory markers had shown no abnormality.

The patient was taken to the operation theatre for bilateral implant removal and total capsulectomy, where implant leakage was found on the left breast and organized hematoma in the retrocapsular area with an intact implant was removed from the right breast, with a total volume of approximately $150 \mathrm{~mL}$ (Figure 3). There were no double capsules as reported in some textured surface implant caused by shear forces between the two surfaces (4).

Bilateral gross capsules were sent for pathology, both showing mild chronic inflammation with stromal fibrosis (Figure 4). Since the implant used was macro-textured type, breast fluid was also sent for CD30 immune staining for breast implant-associated anaplastic large cell lymphoma (BIA-ALCL), which was showed negative results. Implant change was recommended considering the patient's age and for aesthetic purpose; however, the implant was removed according to the patient's request. All incisions were closed primarily, with drains placed in each breast pocket. A quilting suture under the subcutaneous area was done, preventing the formation of excessive seroma or hematoma. Postoperative lab findings showed slight decrease in total protein, which soon recovered before discharge. 

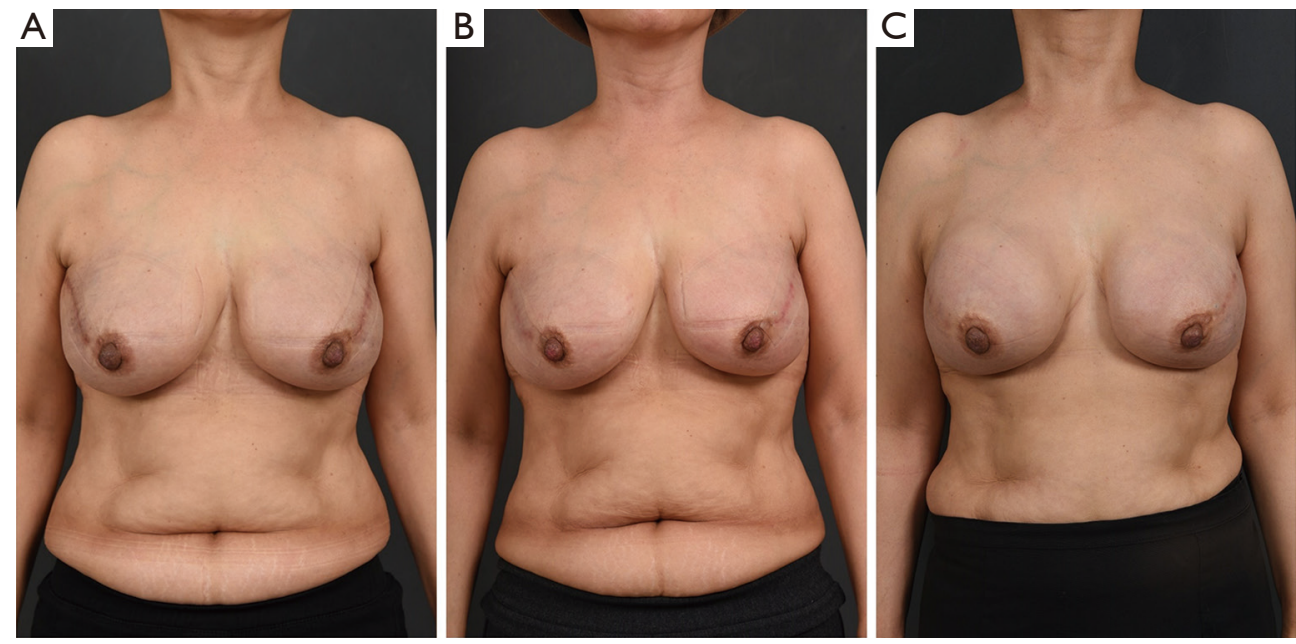

Figure 1 Gross photos of a 54-year-old female patient who underwent bilateral nipple-sparing mastectomy and immediate reconstruction with prepectoral implant insertion. (A) Postoperative 1.5 months gross photo; (B) postoperative 3 months gross photo; (C) postoperative 6 months gross photo indicating apparent sign capsular contracture sign on the right breast.
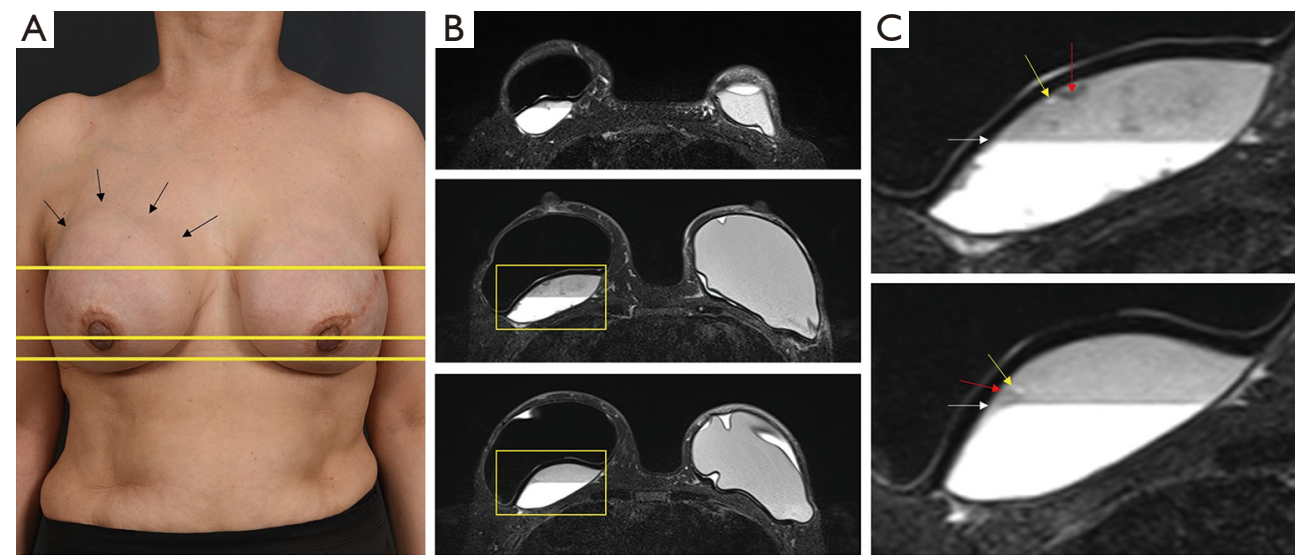

Figure 2 Gross photo and magnetic resonance image of a 54-year-old female patient who underwent bilateral nipple-sparing mastectomy and immediate reconstruction with prepectoral implant insertion. (A) Postoperative 1-year gross photo showing Backer grade III capsular contracture on the right. Yellow line: indicating each level of magnetic resonance imaging from (B). Black arrows indicate capsular contracture. (B) T2 phase magnetic resonance imaging. (C) Close-up of the yellow box area of (B). White arrows: fluid-fluid level indicating hematoma; red arrows: linear air finding beside the vessel; yellow arrows: the vessel originating from the extracapsule area.

\section{Case 2}

A 63-year-old woman with a bilateral subpectoral silicone breast augmentation history approximately 30 years ago was presented to the clinic for severe painless swelling and firmness in the right breast. The swelling was extensive along the medial upper-pole and the lateral inframammary fold area with extension into the axilla (Figure 5). Smoothtype silicone gel prosthesis (Dow Corning Corporation,
$130 \mathrm{cc}$ ) was used in both breasts, resulting in an uneventful painless swelling 10 years after the augmentation. From the beginning of the symptom, the patient often hit her right chest wall to reduce the swelling without any clinical visit. Preoperative magnetic resonance imaging scan showed intracapsular and extracapsular ruptures on the right breast and a bulging intracapsular implant herniation on the left breast within the subpectoral planes bilaterally with no malignancy signs (Figure 5). Overall, the preoperative lab 

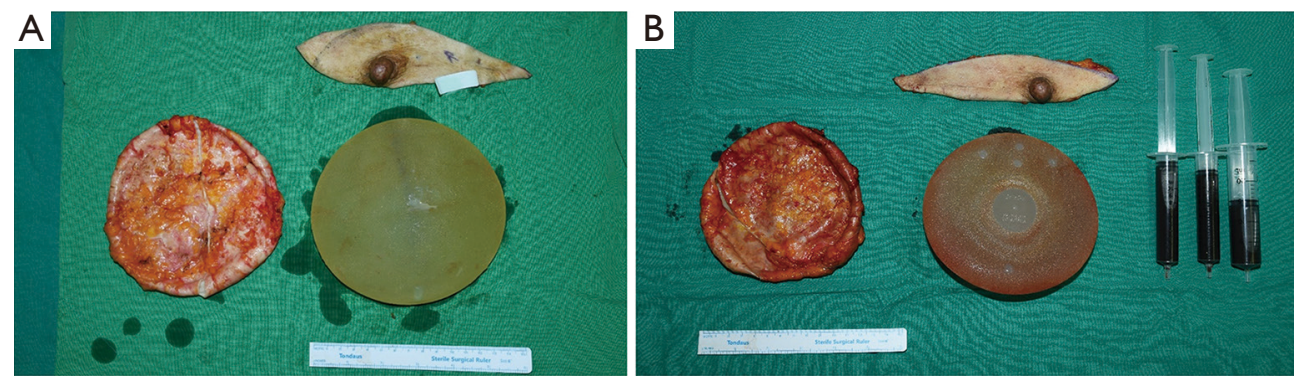

Figure 3 Intraoperative findings of a 54-year-old female. (A) Excised skin, capsule, and implant on the left breast; implant leakage was evaluated on the upper-pole of the anatomically shaped textured implant; (B) excised skin, capsule, and implant on the right breast with evacuated uncoagulated blood from the extracapsular area.
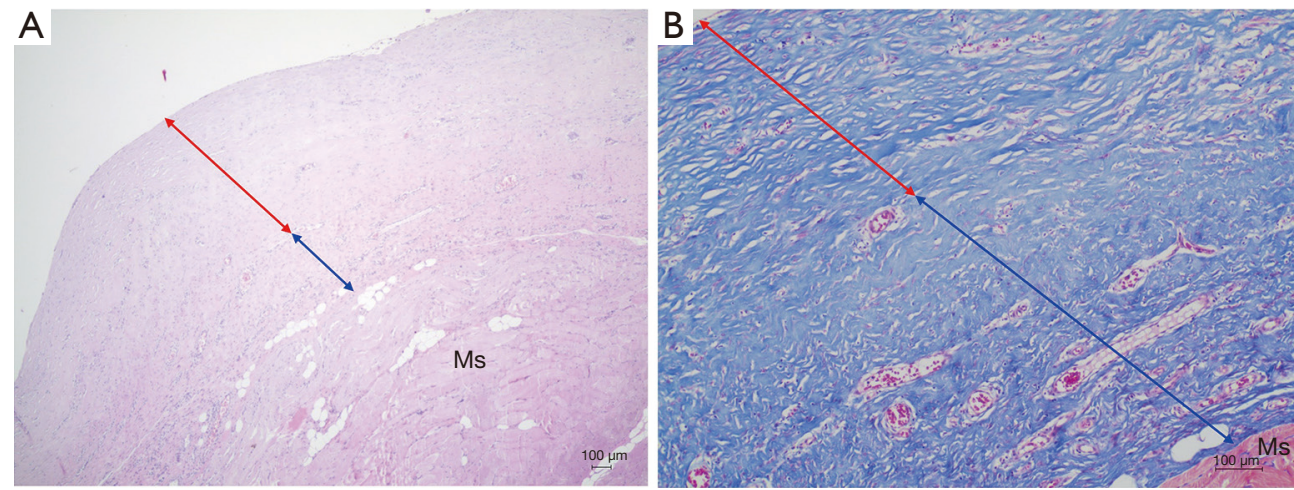

Figure 4 Histological findings from the right excised capsule. (A) Hematoxylin and eosin staining ( $\times 40$ ); (B) Masson's trichrome staining $(\times 100)$. Red arrows indicate acellular dermal matrix; blue arrows indicate vascularized thick capsule, including delayed hematom. Ms, pectoralis major muscle. Scale bar $=100 \mu \mathrm{m}$.
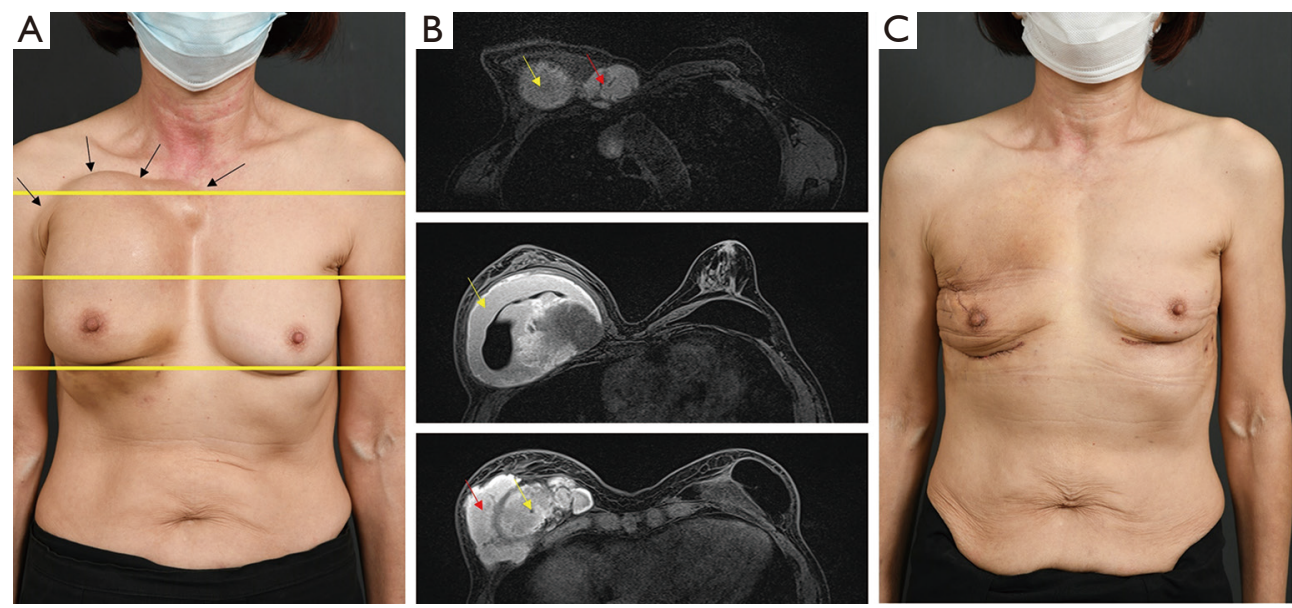

Figure 5 Gross photo and magnetic resonance image of a 63-year-old female patient who underwent bilateral implant augmentation surgery 30 years ago. (A) Preoperative gross photo. Yellow line: each level of magnetic resonance imaging image from b; black arrows: extension from the superior pole to the axilla preoperatively. (B) Preoperative magnetic resonance image. Yellow arrows: intracapsular coagulated hematoma; red arrows: extracapsular hematoma. (C) Postoperative 1-month gross photo. 

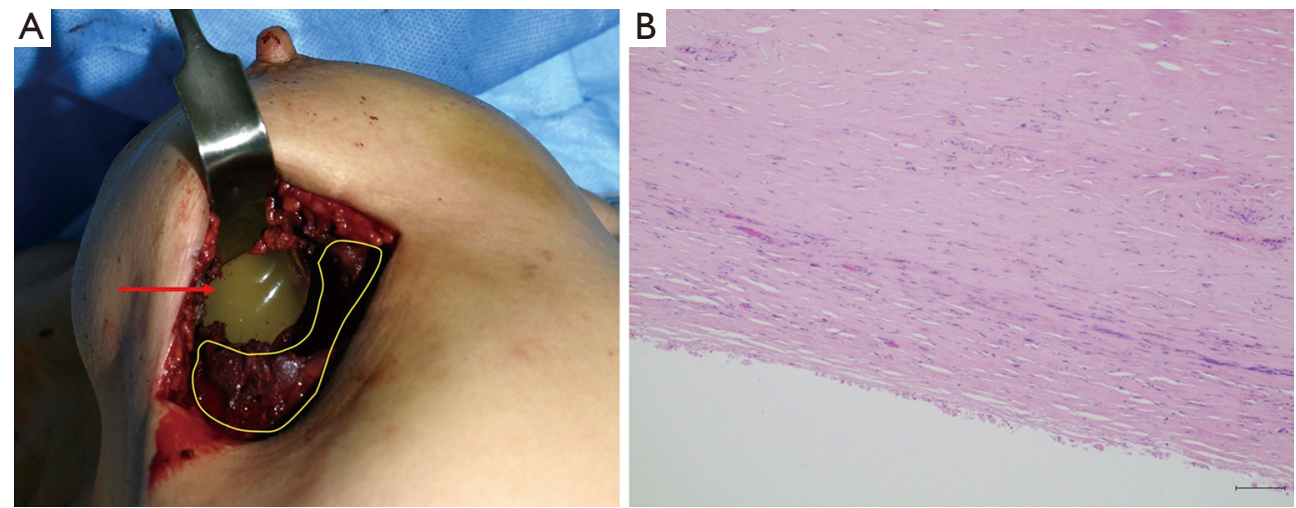

Figure 6 Intraoperative and histologic finding of a 63-year-old female. (A) Incised skin and capsule. Red arrow: intact smooth-type implant; yellow line area: thick coagulated hematoma adhered to the capsule. (B) Hematoxylin and eosin staining showing a fibrous thick capsule. Scale bar $=100 \mu \mathrm{m}$.
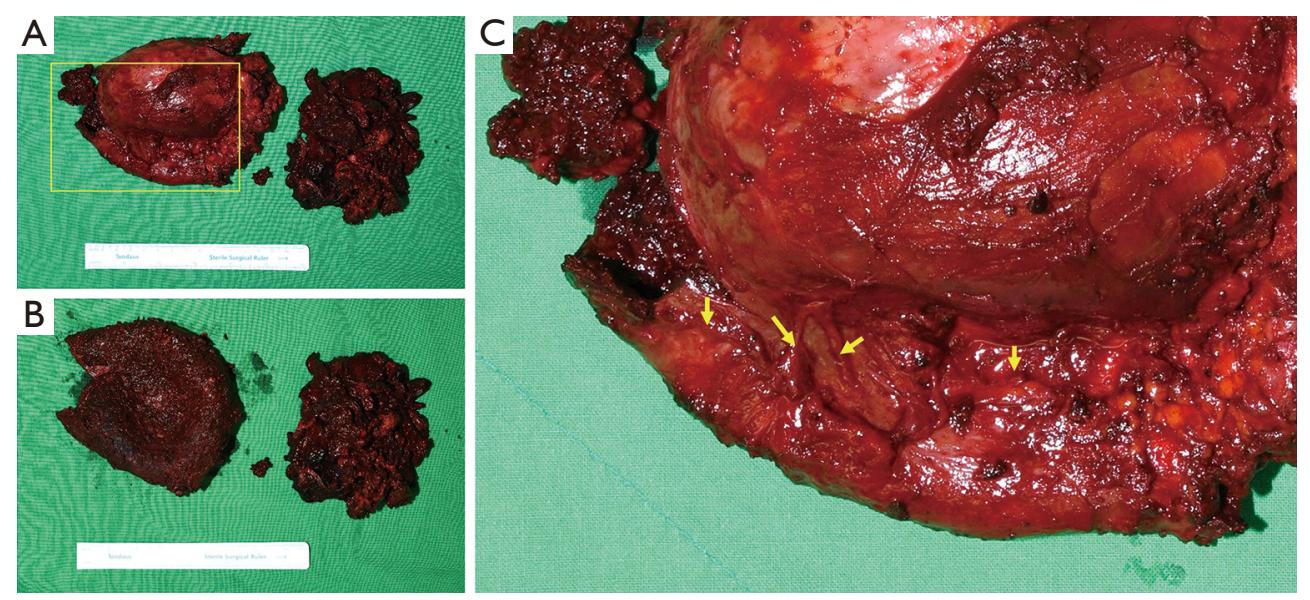

Figure 7 Excised capsule and intracapsular coagulated hematoma from the right breast of a 63-year-old female. (A) Inner side of the capsule and (B) outer side of the capsule; (C) close-up of the yellow line area of (A). Yellow arrows indicate the vessels originating from the capsule, which is presumed to have caused delayed extracapsular hematoma by any erosion incidence.

\section{findings were normal.}

In October 2020, the patient underwent bilateral implant removal with total capsulectomies, draining approximately $300 \mathrm{~mL}$ of semisolidified darkish hematoma from the extracapsular area on the right breast following stab incision from the inferior mammary fold. The capsule, tangled with thick hematoma, was strongly adherent to the surrounding tissues and difficult to excise (Figure 6). The capsule opening presented a large amount of coagulated organized hematoma occupying the interspace between the implant and capsule (Figure 7). The implant inside the capsule was intact. On the left breast, complete implant rupture was found, with sticky-mucous foreign body material extrusion throughout the whole intracapsular area. The specimens were sent for pathology, with results showing a fibrosis from both capsules without malignancy. For assessing the possibility of BIA-ALCL, cytologic specimen and tissue biopsy were performed with negative results. Negative CD30 immune staining for breast fluid ruled out the diagnosis of BIA-ALCL. Increased white blood cell count $(10,310 / \mu \mathrm{L})$ was the only distinctive finding.

All procedures performed in studies involving human participants were in accordance with the ethical standards of the institutional and/or national research committee(s) and with the Helsinki Declaration (as revised in 2013). Written informed consent was obtained from all the patients prior 
to their participation in the study

\section{Discussion}

Since the first case of late hematoma after augmentation mammaplasty reported by Georgiade et al. (5) in 1979 and late capsular hematoma after reconstruction first reported by Marques et al. (6) in 1992, late hematoma development around a breast implant has been described in the literature, which has been observed after reconstructive and aesthetic breast surgery with implants. The delayed post implantation breast hematoma mechanism is not yet well established (7), although previous authors correlated it with direct trauma, clotting disorders, and corticosteroid (8). Capsular contracture, the most common breast implantation complication, has been associated indirectly with late hematoma. Another proposed mechanism for delayed hematoma is bleeding due to capsular microfractures, which might be attributed to the rigidity of the contracted capsule secondary to possible silicone microparticle leakage from the implant. However, in our two presented cases, there were no signs of hematoma or capsular contracture on the side of the breast where silicone leakage was discovered, reporting that a polyurethane-coated implant might evoke an intense, highly vascular inflammation, resulting in late hematoma formation (9). Peters and Fornasier (10) listed several bleeding causes, including the friction between the capsule and an implant fold in the presence of pathologic contracture, friction between the rough surface of a textured implant and the capsule, or infection. Increased capillary permeability due to prolonged inflammation can sustain further bleeding, acting as a stimulant as described for chronic expanding hematomas. In our postimplant reconstruction case, macro-textured implant was inserted in the prepectoral plane. Though there were no gross findings of gross vessel erosion or bleeding point intraoperatively, presumed vascular erosion along the capsule surface may have caused adherent retrocapsular hematoma, and follow-up mammography may have caused the vascular erosion Preoperative capsular contracture, which previous studies described as one of the chain reactions for delayed hematoma, was also observed on the breast where extracapsular hematoma was drained during operation. Polyurethane-covered implants were introduced initially in an effort to decrease the incidence of capsular contracture. However, the polyurethane coating was found to elicit a much more intense foreign body inflammatory response, which is highly vascular and persists for a longer period as a source of pericapsular artery erosion or capsular microfracture. In the augmentation case, capsular contracture appears to have been the primary mechanism for the late hematoma (11), with the excised capsule histologic examination confirming inflammation. Polyurethane coating, as in this case, may have provoked an inflammatory reaction, placing the patient at a higher risk for developing capsular contracture and, possibly, late hematoma. Interestingly, in both of our patients, there were no capsular contracture or hematoma findings in the contralateral breast showing an implant rupture. Hsiao et al. (12) added that the mechanical friction between the rough-textured surface of the prosthesis and the highly vascular capsule may result in an intracapsular hematoma. Textured and smooth surface implants were used in reconstruction and augmentation cases, respectively. In our reconstruction case, preoperative magnetic resonance image showed perivascular linear air findings, although it is not reliable implant rupture signs (13). In our post augmentation, the patient who showed extracapsular and intracapsular hematomas, however, had smooth surface implants, exemplifying the importance of prompt diagnosis and intervention. The delay between documented breast swelling, additional spontaneous external impact, and surgical intervention likely allowed the corresponding hematoma to expand and incorporate. Whenever it is suspected, a late hematoma should be explored and evacuated promptly to prevent possible skin necrosis; otherwise, it could expand and cause severe extent of swelling as demonstrated in our second case. The separation between capsule and the implant is presumed to occur in the interface between the implant surface and the capsule, and the shear forces caused chronic irritation and increased capillary permeability. This is a unique case to an extent where implant degloving caused by mechanical friction has been commonly reported in a textured-type implant, attributing rough surface to the phenomenon. Especially in Asian countries, implant use, conforming to the homogenous acellular dermal matrix development for reconstruction and augmentation, precise detecting and treating associated complications are on demand (14).

As clinical evaluation has limitations, different methods, such as sonography, computed tomography scan, and magnetic resonance, have been adopted. Magnetic resonance image is the ideal method for diagnosing delayed hematoma (15) due to its better specificity in identifying the nature of the collection, including seroma, blood, or clotted hematoma. Thus, in addition to preoperative 
choice of implant and annual ultrasonography, periodical magnetic resonance imaging follow-up from 3 to 5 years interval must be done to prevent spontaneous hematoma regardless of symptoms. Percutaneous needle drainage of fluid collection visualized with imaging procedures is useful to distinguish seroma from hematoma. The aspirate can be sent to pathology to rule out malignancy or infection. In situations of sudden swelling, such as our second case, late hematoma must be highly suspected and the breast must be explored immediately irrelevant of whether it accompanies pain or not. The signs of capsular contracture should not be ignored as it can be the primary origin of late hematoma cascade. Eventually, late hematoma management needs surgical procedure and involves capsulectomy and breast implant replacement or removal (16). The limitation of this case report may be that although we explored and presumed the risk factors of two unusual cases, the exact etiology was not clearly presented.

\section{Conclusions}

Breast prosthesis is popularly implemented widely for aesthetic and reconstructive breast surgeries. As implants come in many shapes, sizes, materials, and surface types, with varying surgical techniques for different purposes, detecting and managing the complications for each type and surgical technique is an essential procedure. Among others, delayed hematoma, especially in Asian patients, remains a rare breast implant procedure complication. Therefore, we report these two rare cases to presume the contributing factors and suggest appropriate treatment with preventions for this complication, which also presents in an asymptomatic character.

\section{Acknowledgments}

Funding: None.

\section{Footnote}

Reporting Checklist: The authors have completed the CARE reporting checklist. Available at http://dx.doi.org/10.21037/ gs-20-854

Conflicts of Interest: All authors have completed the ICMJE uniform disclosure form (available at http://dx.doi. org/10.21037/gs-20-854). The authors have no conflicts of interest to declare.
Ethical Statement: The authors are accountable for all aspects of the work in ensuring that questions related to the accuracy or integrity of any part of the work are appropriately investigated and resolved. All procedures performed in studies involving human participants were in accordance with the ethical standards of the institutional and/or national research committee(s) and with the Helsinki Declaration (as revised in 2013). Written informed consent was obtained from all the patients prior to their participation in the study.

Open Access Statement: This is an Open Access article distributed in accordance with the Creative Commons Attribution-NonCommercial-NoDerivs 4.0 International License (CC BY-NC-ND 4.0), which permits the noncommercial replication and distribution of the article with the strict proviso that no changes or edits are made and the original work is properly cited (including links to both the formal publication through the relevant DOI and the license). See: https://creativecommons.org/licenses/by-nc-nd/4.0/.

\section{References}

1. Collins JB, Verheyden CN. Incidence of breast hematoma after placement of breast prostheses. Plast Reconstr Surg 2012;129:413e-20e.

2. Ibrahim AE, Atiyeh BS. Management of delayed capsular hematoma after breast reconstruction. Aesthetic Plast Surg 2011;35:923-7.

3. Lee JS, Kim JS, Lee JH, et al. Prepectoral breast reconstruction with complete implant coverage using double-crossed acellular dermal matrixs. Gland Surg 2019;8:748-57.

4. Hall-Findlay EJ. Breast implant complication review: double capsules and late seromas. Plast Reconstr Surg 2011;127:56-66.

5. Georgiade NG, Serafin D, Barwick W. Late development of hematoma around a breast implant, necessitating removal. Plast Reconstr Surg 1979;64:708-10.

6. Marques AF, Brenda E, Saldiva PH, et al. Capsular hematoma as a late complication in breast reconstruction with silicone gel prostheses. Plast Reconstr Surg 1992;89:543-5.

7. Kim L, Castel N, Parsa FD. Case of late hematoma after breast augmentation. Arch Plast Surg 2018;45:177-9.

8. Seth AK, Hirsch EM, Kim JY, et al. Hematoma after mastectomy with immediate reconstruction: an analysis of risk factors in 883 patients. Ann Plast Surg 2013;71:20-3. 
9. Grippaudo FR, Renzi L, Costantino B, et al. Late unilateral hematoma after breast reconstruction with implants: case report and literature review. Aesthet Surg J 2013;33:830-4.

10. Peters W, Fornasier V. Late unilateral breast enlargement after insertion of silicone gel implants: A histopathological study. Can J Plast Surg 2007;15:19-28.

11. Hood K, Ganesh Kumar N, Kaoutzanis C, et al. Hematomas in Aesthetic Surgery. Aesthet Surg J 2018;38:1013-25.

12. Hsiao HT, Tung KY, Lin CS. Late hematoma after aesthetic breast augmentation with saline-filled, textured silicone prosthesis. Aesthetic Plast Surg 2002;26:368-71.

13. Gorczyca DP, Gorczyca SM, Gorczyca KL. The diagnosis of silicone breast implant rupture. Plast Reconstr Surg 2007;120:49S-61S.

14. Hvilsom GB, Hölmich LR, Steding-Jessen M, et al. Delayed breast implant reconstruction: a 10year prospective study. J Plast Reconstr Aesthet Surg 2011;64:1466-74.

15. Dean RA, Glener AD, Thomas AB, et al. Silicone Migration and Late Hematoma following Silicone Implant Rupture: Case Report and Literature Review. Plast Reconstr Surg Glob Open 2018;6:e1849.

16. Rogoff H, Marquez JE, Ghosh K, et al. Clinical Outcomes After Hematoma Development: A Study of 627 Tissue Expander Breast Reconstructions. Ann Plast Surg 2020;85:S63-7.
Cite this article as: Lee JH, Hong HK, Kim WH, Kim HJ, Lee J, Park HY, Yang JD, Lee JS. Delayed unilateral hematoma after reconstructive and aesthetic breast surgery with implants in Asian patients: two case reports. Gland Surg 2021;10(4):15151522. doi: $10.21037 /$ gs-20-854 